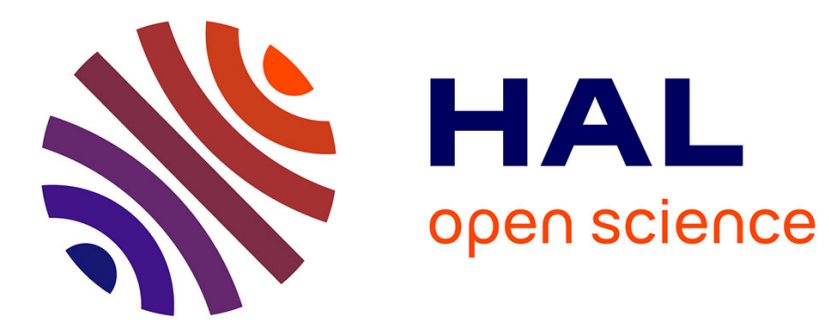

\title{
Ti segregation at CoSi grain boundaries
}

Hannes Zschiesche, Ahmed Charai, Dominique Mangelinck, Claude Alfonso

\section{To cite this version:}

Hannes Zschiesche, Ahmed Charai, Dominique Mangelinck, Claude Alfonso. Ti segregation at CoSi grain boundaries. Microelectronic Engineering, 2019, 203-204, pp.1-5. 10.1016/j.mee.2018.10.009 . hal-02403172

\section{HAL Id: hal-02403172 \\ https://hal.science/hal-02403172}

Submitted on 14 Dec 2020

HAL is a multi-disciplinary open access archive for the deposit and dissemination of scientific research documents, whether they are published or not. The documents may come from teaching and research institutions in France or abroad, or from public or private research centers.
L'archive ouverte pluridisciplinaire HAL, est destinée au dépôt et à la diffusion de documents scientifiques de niveau recherche, publiés ou non, émanant des établissements d'enseignement et de recherche français ou étrangers, des laboratoires publics ou privés. 


\title{
Ti excess at $\mathrm{CoSi}_{2}$ grain boundaries
}

\author{
H. Zschiesche ${ }^{a, *}$, A. Charaï ${ }^{\mathrm{a}}$, D. Mangelinck ${ }^{\mathrm{a}}$, C. Alfonso ${ }^{\mathrm{a}}$ \\ ${ }^{a}$ Aix-Marseille Université, IM2NP, CNRS, Faculté de Saint-Jérôme, Case 142, 13397 \\ Marseille Cedex 20, France
}

\begin{abstract}
The localization of $\mathrm{Ti}$ at grain boundaries after the phase formation of $\mathrm{CoSi}_{2}$ on $\mathrm{Si}(111)$ from a Co-Ti bilayer is examined. For the chemical analysis at the atomic scale, atom probe tomography (APT) is applied. The structure of grain boundaries is analyzed by high resolution transmission electron microscopy (HR TEM). A polycrystalline $\mathrm{CoSi}_{2}$ film has been formed after annealing. Inside the grains of $\mathrm{CoSi}_{2}$, $\mathrm{Ti}$ is determined to be present in low concentration of 0.2 at \%. An excess of $\mathrm{Ti}$ is measured at the grain boundaries. The Ti excess varies for different grain boundaries. HR TEM shows different distances between defects for varying misorientation. The distances between defects are compared with theoretical misfits at the grain boundaries. A relationship between the $\mathrm{Ti}$ excess at grain boundaries determined by APT and the defect densities of grain boundaries analyzed by HRTEM is proposed.
\end{abstract}

Keywords: Cobalt disilicide, $\mathrm{Ti}$ excess at $\mathrm{CoSi}_{2}$ grain boundary, Atom probe tomography, Transmission electron microscopy

\section{Introduction}

$\mathrm{CoSi}_{2}$ films on $\mathrm{Si}$ substrates are widely used in microelectronic applications as contact material beside $\mathrm{TiSi}_{2}$ and NiSi films, depending on the scale. Low electrical resistivity, high thermal stability and the good match of the crystal 5 structure with $\mathrm{Si}$ are the main advantages of $\mathrm{CoSi}_{2}$ [1]. The aim of the intro-

\footnotetext{
${ }^{*}$ Corresponding author: Hannes Zschiesche

Email address: hannes.zschiesche@im2np.fr (H. Zschiesche )
} 
duction of a Ti interlayer between the Co film and the Si substrate was to grow $\mathrm{CoSi}_{2}$ as a single crystal in epitaxy on $\mathrm{Si}(100)$ or $\mathrm{Si}(111)$ by sputtering deposition and reactive diffusion 2 .

The role of the Ti interlayer during the phase formation has been investigated and explanations were proposed. Two possibilities are considered: a) the reduction of native oxide on the silicon substrate b) the formation of a Ti rich phase acting as a diffusion or reaction barrier. Both possibilities should provide homogeneous diffusion and phase formation. The influence of parameters such as the deposition of an alloy instead of a bilayer [3, the substrate orientations interlayer or the Co layer [7] or other elements for the interlayer [8] have been investigated to get a better understanding and a better process. The influence of a Ti capping layer instead of a Ti interlayer was also reported [9, 10]. The $\mathrm{Ti}$ capping layer induced an increase of the nucleation temperature of $\mathrm{CoSi}_{2}$ as well as a preferred texture in comparison to the growth without capping Ti. These effects can be explained by the diffusion of Ti from the capping layer to the interface.

As $\mathrm{Ti}$ is only slightly soluble in $\mathrm{CoSi}_{2}$, it was assumed that $\mathrm{Ti}$ has to be present in the grain boundaries (GBs) after the formation of $\mathrm{CoSi}_{2}$. However, to the best knowledge of the authors, the presence of Ti at the GBs after the formation of $\mathrm{CoSi}_{2}$ has not been shown yet.

Atom probe tomography (APT) enables to detect single atoms and to reconstruct them in 3D volumes with atomic resolution. It allows to quantify tiny excess (number of atoms per unit area 11]) of elements at 2D structures such as GBs or interfaces [12. An excess of elements can origin from strain fields induced by defects [13] as they are present on GBs. The combination of APT and transmission electron microscopy (TEM) is a powerful method to compare excess of elements with structural defects [14].

The aim of this work is the chemical investigation of $\mathrm{CoSi}_{2}$ GBs by APT. The 35 structure at different GBs is analyzed by TEM. A comparison of the determined Ti excess at GBs and the defect density estimated from high resolution 
transmission electron microscopy (HRTEM) investigations will be discussed.

\section{Materials and Methods}

$\mathrm{Si}(111)$ wafers have been immersed in a $5 \%$ dilute $\mathrm{HF}$ for $1 \mathrm{~min}$ to remove

40 native oxide before load in the sputtering system. Successive deposition of $5 \mathrm{~nm}$ Ti and $100 \mathrm{~nm}$ Co from two individual targets of Ti and Co (both $99.99 \%$ purity) has been done by magnetron sputtering. The deposition was performed with a base pressure of about $10^{-8}$ Torr using $99.9999 \%$ pure Ar gas flow of $6.7 \mathrm{sccm}$ at room temperature. Thermal annealing were performed in vacuum

45 up to $850^{\circ} \mathrm{C}$ to fully transform the as-deposited bilayer film into $\mathrm{CoSi}_{2}$.

$\mathrm{X}$-ray diffraction (XRD) measurements (here not shown) performed in the BraggBrentano geometry, with a $1.5^{\circ}$ offset to avoid the signal from the substrate, show the presence of $\mathrm{CoSi}_{2}$ with a strong peak of the (111) orientation and minor peaks of further orientations. TEM lamellas and APT tips have been

50 prepared using a focused ion beam (FIB) FEI Helios 600. APT measurements were performed on a LEAP $3000 \mathrm{HXR}$. Voltage mode at $40 \mathrm{~K}, 200 \mathrm{kHz}, 20$ $\%$ pulse fraction and $0.2 \%$ detection rate was applied in order to get optimal information in the compromise of resolution and volume size. IVAS software was used for the reconstruction following the common procedure [15, 16]. TEM has been performed on a TECNAÏ G20 for microstructure investigations and energy dispersive X-ray spectroscopy (EDS) analysis and on a $\mathrm{C}_{\mathrm{s}}$-corrected TITAN 80-300 equipped with a GIF Tridiem 863. The acceleration voltage was always chosen to be $200 \mathrm{kV}$.

\section{Results}

60 3.1. $A P T$

Figure 1 presents three APT volumes which contain each one GB. The presence of the GB is indicated by an increased Ti concentration along a flat plane, as highlighted in figure 1 by the isoconcentration surface for Ti 1.5 at \%. In order to determine the amount of $\mathrm{Ti}$ at the GBs, cylinders with a diameter of 


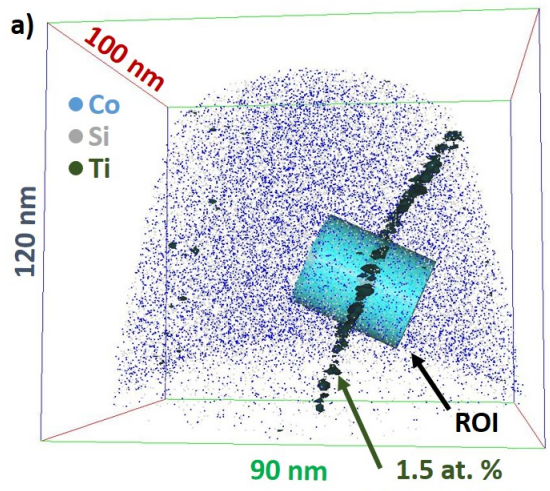

b)
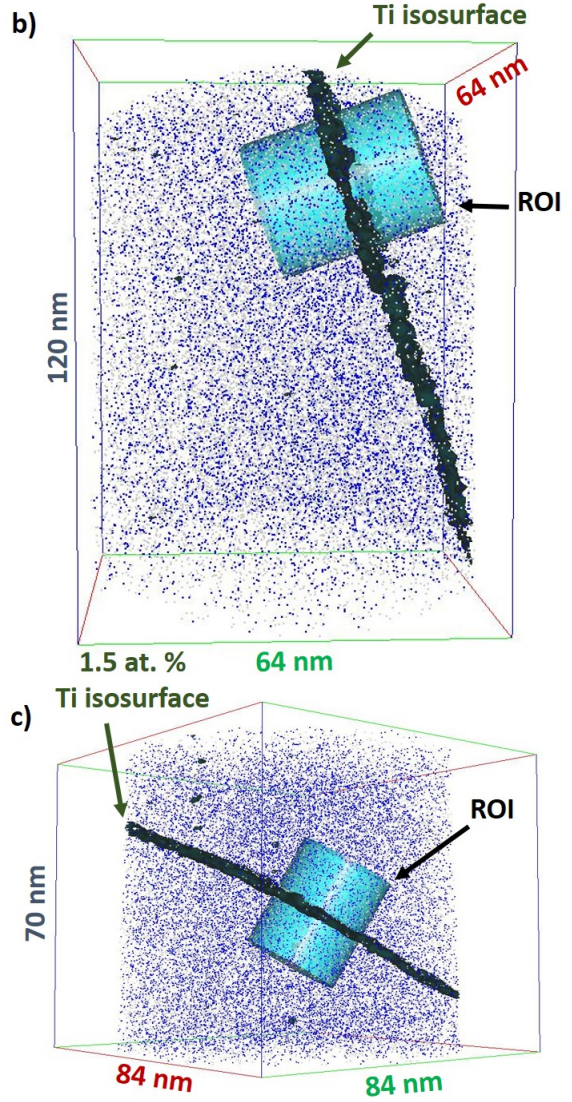

Figure 1: APT volumes of $\mathrm{CoSi}_{2}$ contain GBs with excess of $\mathrm{Ti}$ (1.5 at. \% isosurface highlighted). a) GB 1 b) GB 2 c) GB 3 
$25 \mathrm{~nm}$ have been placed with their z-axis along the normal of the GB plane as shown in figure 1. The 1d concentration profile has been measured along the $\mathrm{z}$-axis of these cylinders as shown in figure $2 \mathrm{a}$ for the cylinder in figure $1 \mathrm{a}$. A low constant concentration can be seen inside the grains. At the GB, a sharp peak of $\mathrm{Ti}$ indicates the increased concentration of $\mathrm{Ti}$. The full width at half maximum (FWHM) of the peak is about $1.5 \mathrm{~nm}$.
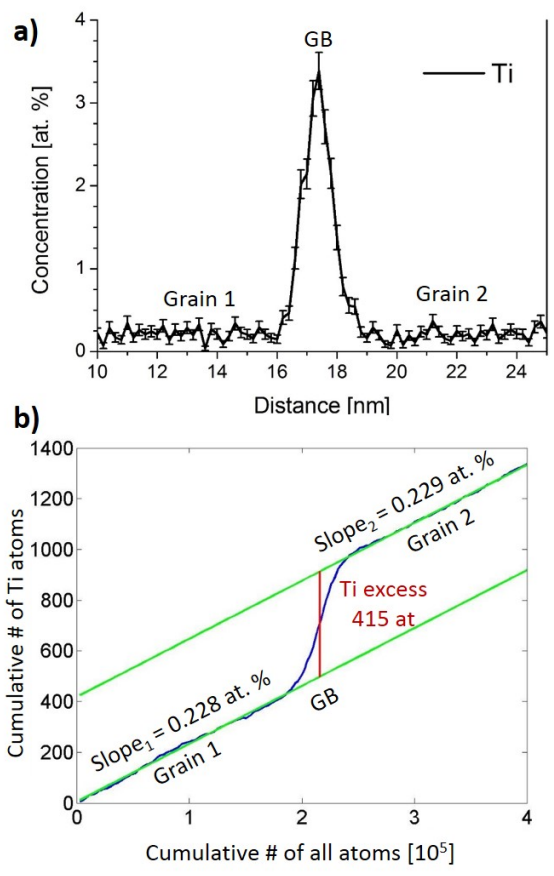

Figure 2: a) The concentration profile along the z-axis of the cylinder in figure 1 a visualizes the increased concentration of $\mathrm{Ti}$ at the GB. b) The constant concentration inside the grains can be fitted with a linear function in the integral profile. The slope corresponds to the concentration (about 0.2 at. \% for both grains). The step height at the GB is related to the number of $\mathrm{Ti}$ atoms on the base area of the cylinder (415 at).

70

However, the comparison of the excess concentration at a GB is only semiquantitative. The spatial resolution in the APT is not isotropic and thus experimental broadening due to different spatial resolution can occur depending on the orientation of the grain boundary inside the APT tip volume. This can 
change the local excess concentration of identical grain boundaries. In contrast, the determination of the excess number of atoms projected on the GB plane allows a more quantitative comparison as it is not affected by anisotropic effects [11. The value of the Ti excess at the GB can be specified as the number of atoms per unit area. Thus, experimental broadening of the GB plane has no influence on the excess quantity.

The accumulation of $\mathrm{Ti}$ atoms over the accumulation of all atoms along the $\mathrm{z}$-axis of the cylinder is shown in the integral profile (Figure 2b). For a constant concentration, as seen in the grains, a linear behavior is verified: the concentration is determined from the slope of the fit. The Ti concentration in the grains 85 is about 0.2 at \%. The offset between the two slopes of the grains at the GB corresponds to the Ti excess at the analyzed GB area (red line). In this example it is determined to 415 atoms integrated over the base area of the cylinder. Dividing the number of atoms by the base area of the cylinder gives the value of Ti excess at the GB per unit area. The same procedure was applied for the three different GBs from figure 1 and the Ti excess is visualized in figure 3 . The Ti excess is significantly different for the three investigated GBs.

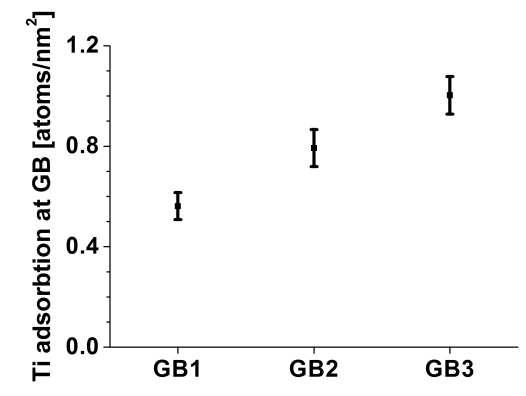

Figure 3: The comparison of Ti excess at different GBs shows a significant variation.

\subsection{TEM}

Figure 4 shows a low angle tilt GB. The fast Fourier transformations (FFT) (inset in figure 4 4 ) demonstrates that both adjacent grains are in [10-1] zone 


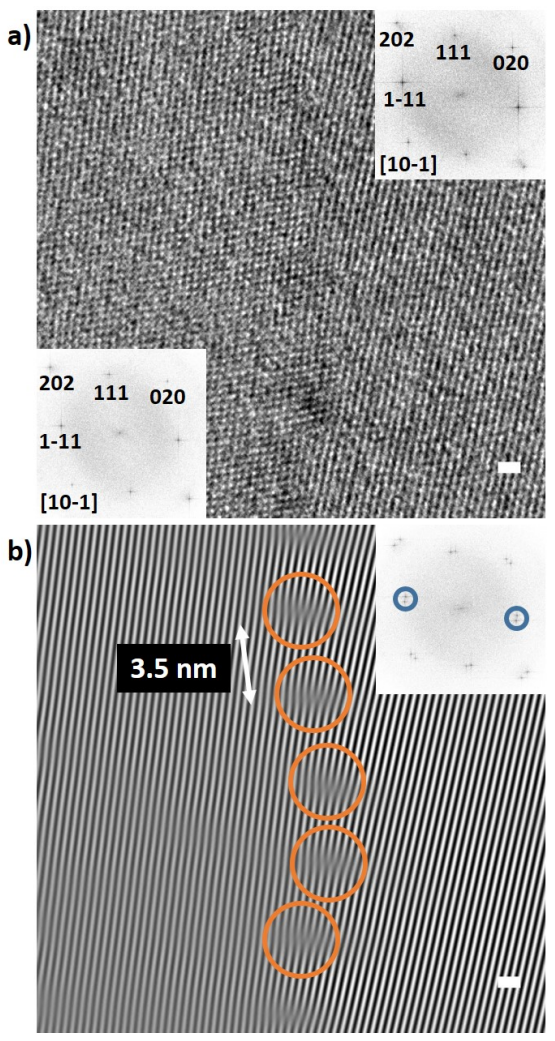

Figure 4: HREM on low angle tilt GB. a) Two grains in [10-1] zone axis are slightly tilted around the [10-1] axis to each other (about $5^{\circ}$ ). b) Bragg filtering of (1-11) planes visualizes the misorientation of the grains. Furthermore, defects along the GB becomes visible.

axis and thus have a close relationship. A rotation of about $5^{\circ}$ around the $[10-$ 1] axis can be measured in the FFT of a region including both grains, where two spots are found close to each other for all directions (inset figure $4 \mathrm{~b}$ ). A rotation axis parallel to GB plane, as in this example, identifies a tilt GB. Different intensities of spots for same directions of the both grains indicate that an additional misorientation is present. However, due to the fact that both grains are in the same zone axis, the total misorientation is assumed to be low and the GB has a main low angle tilt character.

Figure $4 \mathrm{~b}$ is an image obtained only from the (1-11) spots of the FFT. It shows the (1-11) planes of $\mathrm{CoSi}_{2}$. This Bragg-filtered image of selected planes, 


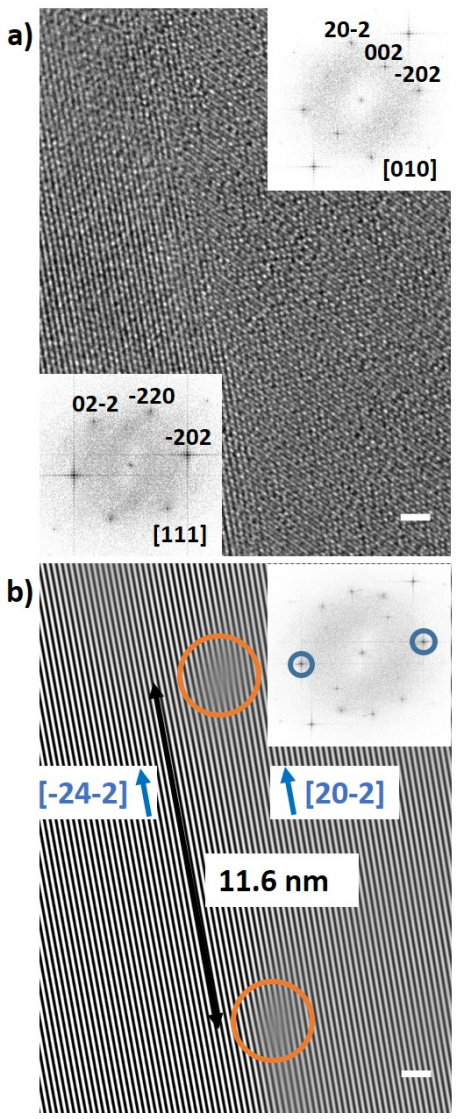

Figure 5: HREM on special GB. a) Two grains in [111] and [010] zone axis are visible. The misorientation of the two zones is $54.7^{\circ}$. Both grains have the $(\overline{202})$ planes in common. b) Bragg filtering of the (202) planes shows defects at the GB in very large distance (about $11.6 \mathrm{~nm}$ ) compared to figure 4

visualizes defects along the GB. They show a periodic distance of about $3.5 \mathrm{~nm}$.

Figure 5 is an example for a special twist GB [17. The two adjacent grains are in different low indexed zone axis ([111] and [010], compare FFTs of insets in figure 5. They have the $(\overline{2} 02)$ planes in common. Thus, the rotation axis is perpendicular to the $(\overline{2} 02)$ planes. Furthermore, the GB plane is aligned close 110 to the $(\overline{2} 02)$ planes and so the rotation axis is almost perpendicular to the GB plane. That identifies a main twist GB character. The misorientation of the two zone axis is $54.7^{\circ}$. Figure 5 b is the Bragg-filtered image of the $(\overline{202})$ planes. 
Defects in the $(\overline{2} 02)$ planes can be found and are highlighted. The distance is about $11.6 \mathrm{~nm}$. Their origin is in the mismatch of the planes perpendicular to the GB plane. In parallel to the viewing direction these are the $(\overline{2} 4 \overline{2})$ planes for the grain on the left side of the figure and the $(20 \overline{2})$ planes for the grain on the right side of the figure (normal vectors of planes indicated in figure $5 \mathrm{~b}$ ).

EDS, energy-filtered transmission electron microscopy (EFTEM) and scanning transmission electron microscopy (STEM) electron energy loss spectroscopy (EELS) have been applied in order to investigate the presence of $\mathrm{Ti}$ at the GBs directly in TEM (not shown). However, no Ti excess was detected at different GBs. Since the APT results of section 3.1 show a systematic enrichment of $\mathrm{Ti}$ on GBs, the local concentration variation of $\mathrm{Ti}$ should be under the detection limit of these analytical TEM techniques and the combination of TEM and APT is crucial for this investigation.

\section{Discussion}

The values of $\mathrm{Ti}$ excess at $\mathrm{CoSi}_{2}$ GBs investigated by APT determine a number of atoms per unit area. In the Gibbs approach, it is assumed that all the excess atoms are located on the GB plane with no thickness. The sharp peak at the GB in the concentration profile of figure 2 a justifies that assumption. The FWHM is about $1.5 \mathrm{~nm}$ and can be explained by experimental broadening. Furthermore, the defects in the GB plane can be energetically favorable positions for Ti atoms, as seen for other material system with excess on defects [13. Thus, it is of interest to estimate a defect density per unit area on the GBs from 135 the TEM investigations and compare them with the Ti excess.

Figure 4 shows that the distance between the defects along a low angle GB plane is about $d_{d i s l o}=3.5 \mathrm{~nm}$. Low angle tilt GBs are well described by edge dislocations parallel to the rotation axis [18]. Their distance $d_{d i s l o}$ is geometrical predicted from the Read and Shockley expression $d_{\text {dislo }}=b / \Theta$ where $b$ is the norm of the Burgers vector and $\Theta$ is the misorientation of the GB [19. With the measurement of the distance between the defects and the misorientation 


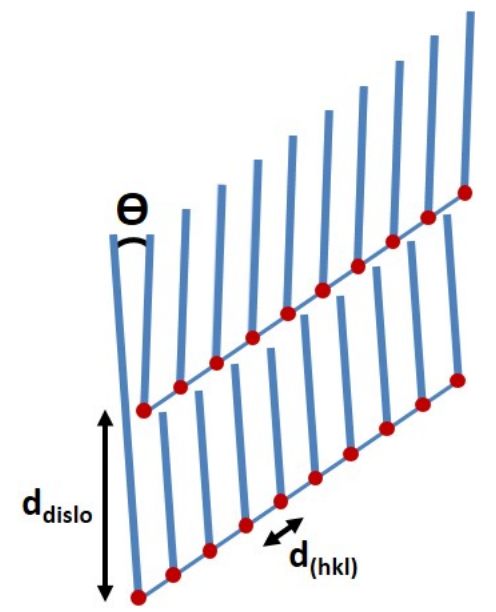

Figure 6: Schematic drawing of defect density calculation on low angle tilt GB with edge dislocations paralle to the rotation axis.

of the GB by HRTEM, the norm of the Burgers vector can be calculated to $b=0.31 \mathrm{~nm}$, even if the quality of the HRTEM image is not sufficient for a direct determination. This value is in the order of the lattice plane distances of $\mathrm{CoSi}_{2}(1 \overline{1} 1)$ planes. Thus, it can be assumed that there are primary $\vec{b}=\mathrm{a}(1 \overline{1} 1)$ edge dislocations at the $[10 \overline{1}]$ low angle tilt GB. For verification it is necessary to determine the orientation of the Burgers vector in a HRTEM image. Modeling the low angle tilt GB by the superposition of supercells in [101] orientation and $5^{\circ}$ misorientation verifies a distance of atomic positions in coincidence of about $150 \quad 3.5 \mathrm{~nm}$.

Additional planes from edge dislocations are normal to the plane of the HRTEM image. Their distance is identical to the atomic spacing between the lattice planes in the viewing direction $[10 \overline{1}]\left(d_{(10 \overline{1})}=0.378 \mathrm{~nm}\right)$. From that, the unit area for one defect is calculated to $d_{(10 \overline{1})} \times d_{\text {dislo }}=1.33 \mathrm{~nm}^{2}$ (compare scheme in figure 6). If every defect core is occupied by one $\mathrm{Ti}$ atom, an $\mathrm{Ti}$ excess of about 0.75 atoms $/ \mathrm{nm}^{2}$ is expected for the investigated low angle GB. This estimation of the excess value is in between the two Ti excesses determined by APT at the GB1 and GB2 (Figure 3). 


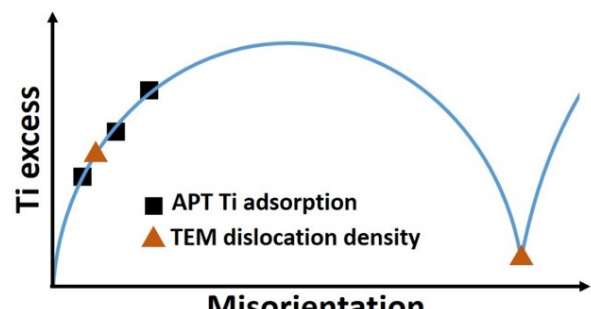

Figure 7: Schematic drawing of Ti excess over misorientation compared with estimated GB defect density.

In figure 7, a schematic model of the relation between the Ti excess at the GB and their misorientation is drawn. As it is assumed that the Ti excess is correlated to the defect density, it is expected to be minimal for very low misorientation and at special GBs where the lattices of both adjacent grains are in high coincidence. From the calculation above, the Ti excess of GB1 and GB2 investigated by APT are slightly smaller and bigger than the defect density at the low angle GB investigated in TEM. It can be estimated that they are in the same misorientation region, as well as GB3.

The defects in the special GB (Figure 5 ) have a distance 3 times larger than the defects in the presented low angle GB. Thus, a significant lower defect density and by this Ti excess at the GB would be expected to be measured. The measured defect distance can be verified by comparison of the distance between coincidence sites of the different planes parallel to the viewing direction perpendicular to the GB. Their normal vectors are drawn in figure 5 b. For the grain on the left in [111] viewing direction it corresponds to the atomic spacing between $(\overline{2} 4 \overline{2})$ planes $d_{(\overline{2} 4 \overline{2})}=2.187 \mathrm{~nm}$. For the grain on the right in [010] viewing direction it corresponds to the atomic spacing between $(20 \overline{2})$ planes $d_{(20 \overline{2})}=1.894 \mathrm{~nm}$. Distances between defects along the GB are estimated to be at minimums of the differences in multiple atomic distances which are coincident sites for differences close to 0 . After 7 and 6 atomic distances, respectively, the difference has a minimum. Thus, the distance of defects is estimated to be about $13.2 \mathrm{~nm}$, that is in the order of the measured distance. The $(\overline{2} 4 \overline{2})$ planes 
are not resolved, thus a simultaneous Bragg-filtering of $(\overline{2} 4 \overline{2})$ and $(202)$ planes is not possible.

However, strong assumptions have been made to calculate the defect density on the GB plane from HRTEM image. The number of Ti atoms localized per defect might be also different from one. In addition, different kinds of defects (edge, screw or mixed dislocations, steps...) may have different behavior in view on Ti excess. Simulations could help to compare the proposed relationship to theoretical thermodynamic results.

To improve the interpretation of the presented results, a precise correlation of the Ti excess to the GB misorientation and the GB plane would be necessary. In principal, acquired APT volumes can contain enough crystallographic information to determine the misorientation through the density variation along the crystallographic poles and lattice planes [20, 21]. Nevertheless, obtaining this information is difficult and usually works easier for pure metals [20, 21]. Moreover, there are limitations if the GB is at the border of the APT volume. In the volumes shown in figure $3 \mathrm{~b}$ and 3 , there is not enough information to determine the misorientation properly. Another method to determine the misorientation of the GB is the use of electron microscopy before the APT run. Indeed, orientation mapping of the tip by transmission Kikuchi diffraction (TKD) in a scanning electron microscope (SEM) or by nanobeam diffraction in a TEM has shown to be promising for systematic investigations between misorientation and excess at GBs [22, 23].

Further studies are in progress to get better correlation between the misorientation and the $\mathrm{Ti}$ excess as well as correlation between the structure of $\mathrm{CoSi}_{2}$ GBs and the Ti excess.

\section{Conclusion}

In conclusion, $\mathrm{Ti}$ segregates to grain boundaries of $\mathrm{CoSi}_{2}$. The amount of excess depends on the grain boundary characteristic as misorientation and defect structure. A relationship between defects and Ti localization is proposed. It 
as well as by an exhaustive study of correlation between misorientation and $\mathrm{Ti}$ excess.

\section{Acknowledgment}

This work was supported by the French government through the program

¿ [1] S. P. Murarka, Intermetallics 3 (1995) 173-186. doi 10.1016/ 0966-9795(95) 98929-3.

[2] M. L. A. Dass, D. B. Fraser, C.-S. Wei, Appl.\Phys.\Lett. 58 (1991) 1308. doi: $10.1063 / 1.104345$.

225

[3] S. L. Hsia, T. Y. Tan, P. Smith, G. E. McGuire, J. Appl. Phys. 70 (1991) 7579-7587. doi:10.1063/1.349713.

[4] P. Liu, B. Z. Li, Z. Sun, Z. G. Gu, W. N. Huang, Z. Y. Zhou, R. S. Ni, C. L. Lin, S. C. Zou, F. Hong, G. A. Rozgonyi, J. Appl. Phys. 74 (1993) 1700-1706. doi:10.1063/1.354824.

230

[5] T. I. Selinder, T. A. Roberts, D. J. Miller, M. A. Beno, G. S. Knapp, K. E. Gray, S. Ogawa, J. A. Fair, D. B. Fraser, J. Appl. Phys. 77

口 (1995) 6730. URL: http://ieeexplore.ieee.org/articleDetails.jsp? arnumber=5151061, doi $10.1063 / 1.359091$. 
[6] F. Hong, G. A. Rozgonyi, B. K. Patnaik, Appl. Phys. Lett. 64 (1994) 2241-

235

[7] A. Vantomme, M. A. Nicolet, N. D. Theodore, J. Appl. Phys. 75 (1994) 3882-3891. doi $10.1063 / 1.356033$

[8] C. Detavernier, R. L. Van Meirhaeghe, F. Cardon, K. Maex, H. Bender,

240 B. Brijs, W. Vandervorst, J. Appl. Phys. 89 (2001) 2146-2150. doi 10. 1063/1.1340598.

[9] C. Detavernier, R. L. Van Meirhaeghe, F. Cardon, R. A. Donaton, K. Maex,

घ Microelectron. Eng. 50 (2000) 125-132. doi 10.1016/S0167-9317(99) 00272-5.

[10] C. Detavernier, R. L. Van Meirhaeghe, W. Vandervorst, K. Maex, Microelectron. Eng. 71 (2004) 252-261. doi 10.1016/j.mee.2003.12.002.

[11] B. W. Krakauer, D. N. Seidman, Phys. Rev. B 48 (1993) 6724-6727. doi:10. 1103/PhysRevB.48.6724.

[12] M. A. Khan, S. P. Ringer, R. Zheng, Adv. Mater. Interfaces 3 (2016). doi $10.1002 /$ admi.201500713

[13] A. Kwiatkowski da Silva, G. Leyson, M. Kuzmina, D. Ponge, M. Herbig, S. Sandlöbes, B. Gault, J. Neugebauer, D. Raabe, Acta Mater. 124 (2017) 305-315. doi $10.1016 / \mathrm{j}$.actamat.2016.11.013.

[14] A. Stoffers, J. Barthel, C. H. Liebscher, B. Gault, O. Cojocaru-Mirédin, 255 ¿ C. Scheu, D. Raabe, Microsc. Microanal. 23 (2017) 291-299. doi 10.1017/ S1431927617000034.

[15] P. Bas, A. Bostel, B. Deconihout, D. Blavette, Appl. Surf. Sci. 87-88 (1995) 298-304. doi $10.1016 / 0169-4332(94) 00561-3$.

[16] F. Vurpillot, B. Gault, B. P. Geiser, D. J. Larson, Ultramicroscopy 132 (2013) 19-30. doi 10.1016/j.ultramic.2013.03.010. 
[17] V. Randle, Scr. Mater. 54 (2006) 1011-1015. doi 10.1016/j.scriptamat. 2005.11 .050

[18] L. Prieseter, Grain boundaries from theory to engineering, 2012. doi 10. 1007/978-3-642-33848-9.

265

[20] M. P. Moody, F. Tang, B. Gault, S. P. Ringer, J. M. Cairney, Ultramicroscopy 111 (2011) 493-499. URL: http://dx.doi.org/10.1016/j. ultramic.2010.11.014. doi 10.1016/j.ultramic.2010.11.014.

[21] L. Yao, M. P. Moody, J. M. Cairney, D. Haley, A. V. Ceguerra, C. Zhu, S. P. 【 Ringer, Ultramicroscopy 111 (2011) 458-463. URL: http://dx.doi.org/

a 10.1016/j.ultramic.2010.11.018 doi $10.1016 / j . u l t r a m i c .2010 .11$. 018.

275 [22] K. Babinsky, W. Knabl, A. Lorich, R. De Kloe, H. Clemens, S. Primig, 1 Ultramicroscopy 159 (2015) 445-451. URL: http://dx.doi.org/10.1016/ j.ultramic.2015.05.014. doi 10.1016/j.ultramic.2015.05.014.

[23] M. Herbig, D. Raabe, Y. J. Li, P. Choi, S. Zaefferer, S. Goto, Phys. Rev. Lett. 112 (2013) 1-5. doi 10.1103/PhysRevLett.112.126103. 